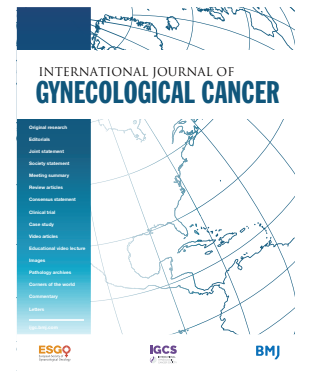

${ }^{1}$ Radiation Oncology, Duke Cancer Institute, Durham, North Carolina, USA

${ }^{2}$ Gynecologic Oncology, Duke Cancer Institute, Durham, North Carolina, USA

\section{Correspondence to}

Dr Junzo Chino, Duke Cancer Institute, DUMC 3085, Durham, North Carolina 27710, USA; junzo.chino@duke.edu

Received 3 September 2021 Accepted 15 October 2021

\title{
Role of stereotactic body radiotherapy in gynecologic radiation oncology
}

\author{
Rachel Shenker, ${ }^{1}$ Sarah J Stephens, ${ }^{1}$ Brittany Davidson, ${ }^{2}$ Junzo Chino (D) ${ }^{1}$
}

\begin{abstract}
Stereotactic body radiotherapy (SBRT, also referred to as stereotactic ablative radiotherapy (SABR)) has been used in the treatment of primary and metastatic solid tumors, and increasingly so in gynecologic oncology. This review article aims to summarize the current literature describing the utility of SBRT in the primary, recurrent, and limited metastatic settings for gynecologic malignancies. The use of SBRT in both retrospective and prospective reports has been associated with adequate control of the treated site, particularly in the setting of oligometastatic disease. It is not, however, recommended as an alternative to brachytherapy for intact disease unless all efforts to use brachytherapy are exhausted. While phase I and II trials have established the relative safety and potential toxicities of SBRT, there remains a dearth of phase III randomized evidence, including the use of immunotherapy, in order to better establish the role of this technique as a method of improving more global outcomes for our patients with gynecologic cancers.
\end{abstract}

\section{FUNDAMENTAL BASIS FOR STEREOTACTIC BODY RADIOTHERAPY (SBRT)}

Radiation therapy is commonly integrated into combined modality treatment for many gynecologic cancers. In the modern era, the use of stereotactic body radiotherapy (SBRT, also referred to as stereotactic ablative radiotherapy (SABR)) has been used in a variety of solid tumors, and increasingly so in gynecologic oncology. By definition, SBRT consists of precisely delivered radiation in large dose fractions in 1-5 treatments, with limited dose delivered to the surrounding normal tissue. The first common use of stereotactic irradiation was in the context of intracranial disease (stereotactic radiosurgery), and the use of this technique was adapted extracranially as SBRT. ${ }^{1}$ The first uses of SBRT in the extracranial setting occurred in Japan in the late 1990s, gaining traction in the USA and Europe in the early 2000s. ${ }^{2}$ Since then, this technique has been adapted for the use of a wide array of clinical scenarios for both primary and metastatic tumors.

The basic principles of SBRT lie within radiobiology and physics. In order to achieve sufficient cellular injury to targets and spare normal tissue, there must be a fine balance of dose distribution and amount of radiation per treatment. Classically, radiation therapy is given in smaller fractions (1.8-2Gy per fraction) over the course of 20-35 daily treatments, in order to maximize the differential ability of normal tissue to repair sub-lethal damage while still causing malignant cell death. SBRT, in contrast, reduces this to 1-5 total treatments, ranging from $24 \mathrm{~Gy}$ in a single fraction to 60 Gy in $3-5$ fractions. These fractionation schemes vary depending on the goals of treatment, neighboring normal tissues, and provider/institutional protocols; however, all of these schedules aim to achieve biologic effective doses (BED) sufficient to control targets and remain below the threshold for normal tissue injury. Malignant and fast-growing tissues are less sensitive to fraction size, while most normal tissues are more sensitive. This is the historical rationale for why larger fraction sizes have been avoided, particularly when treating in close proximity to sensitive tissues.

This paradigm can be circumvented by more accurate radiation delivery techniques, both in the precision of how the treatment volume may be shaped to fit a given target (such as with the use of techniques such as intensity-modulated radiotherapy and volumetricmodulated arc therapy techniques), but also in the precision of imaging at the time of treatment delivery and patient immobilization. Many modern linear accelerators include 'on-board' imaging capabilities, which can confirm that the targets of treatment are encompassed by the irradiated volume while simultaneously ensuring that normal tissues are excluded (Figure 1). This imaging can be performed on a per-fraction basis, such that day-to-day variations in patient set-up and organ motion can be accounted for (Figure 2). When planning for SBRT, patient set-up includes an immobilization device that minimizes potential set-up errors during treatment delivery. This is coupled with evaluation of whether the patient can lie still during treatment, a crucial component when assessing patients in clinic for potential SBRT. There are also means of tracking and/or limiting respiratory motion, such that targets within mobile structures such as the lung and liver can be treated with much smaller margins. The combination of these two technical advancementsmore conformal treatment plans and more accurate delivery-allows for increased sparing of normal tissues compared with that typically seen with standard fractionation (Figure 3).

In this review article we discuss the current and future directions of the use of SBRT in primary, recurrent, and oligometastatic gynecologic malignancies. 


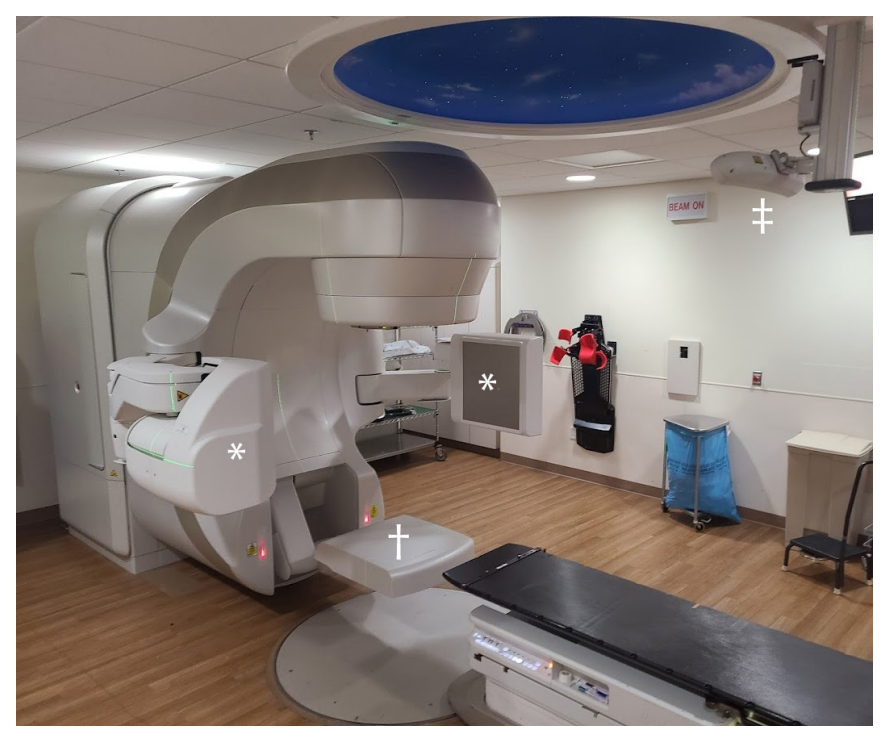

Figure 1 A modern linear accelerator with on-board imaging arms deployed. Opposite the treatment head, conventional 'portal' imaging of the treatment beam can be obtained with a mega-voltage detector panel $(\dagger)$. On the sides of the accelerator, a paired kilo-voltage X-ray source and detector panel (*) are oriented orthogonal to the treatment head. These can obtain plain films, fluoroscopic images and, when rotated $360^{\circ}$, a cone-beam computed tomography (CBCT) image for volumetric imaging of the patient in the treatment position. A ceiling mounted infrared camera ( $\ddagger)$ can track respiratory motions of the chest wall to account for internal movement of targets and normal tissues.

\section{SBRT IN THE MANAGEMENT OF PRIMARY AND LOCALLY RECURRENT DISEASE}

Initial localized treatment of cervical cancers generally includes the use of brachytherapy boost after external beam. The role of SBRT in lieu of brachytherapy for those who have relative contraindications or lack of access to the procedure is an important topic of investigation. $^{3}$

There have been few studies that have reviewed outcomes of patients who received SBRT boost in lieu of brachytherapy boost for a primary gynecologic malignancy (Table 1). A retrospective study including 25 patients with newly diagnosed cervical cancer treated with external beam radiotherapy followed by SBRT boost reported a 3 -year local control rate of $80.9 \%$ with $20 \%$ of patients experiencing grade 3 toxicity including hematuria and hematochezia. ${ }^{4}$ Patients included in this analysis were unable to undergo brachytherapy boost due to either anatomic issues (ie, narrow vagina or cervical stenosis), tumor size felt to be too large for adequate brachytherapy dose distribution, morbid obesity, or other medical co-morbidities precluding brachytherapy. The authors of this study conclude that although SBRT may not replace brachytherapy, SBRT may be a reasonable alternative if a patient is unsuitable for brachytherapy or if the procedure is not available. However, it is notable that there have been recent advances in the techniques in brachytherapy as well. Image-guided brachytherapy using three-dimensional imaging such as magnetic resonance imaging (MRI) for planning allows for highly precise and individualized treatment. A recent report from the multi-institutional prospective EMBRACE study of 1426 women using these techniques found a local control of $92 \%$

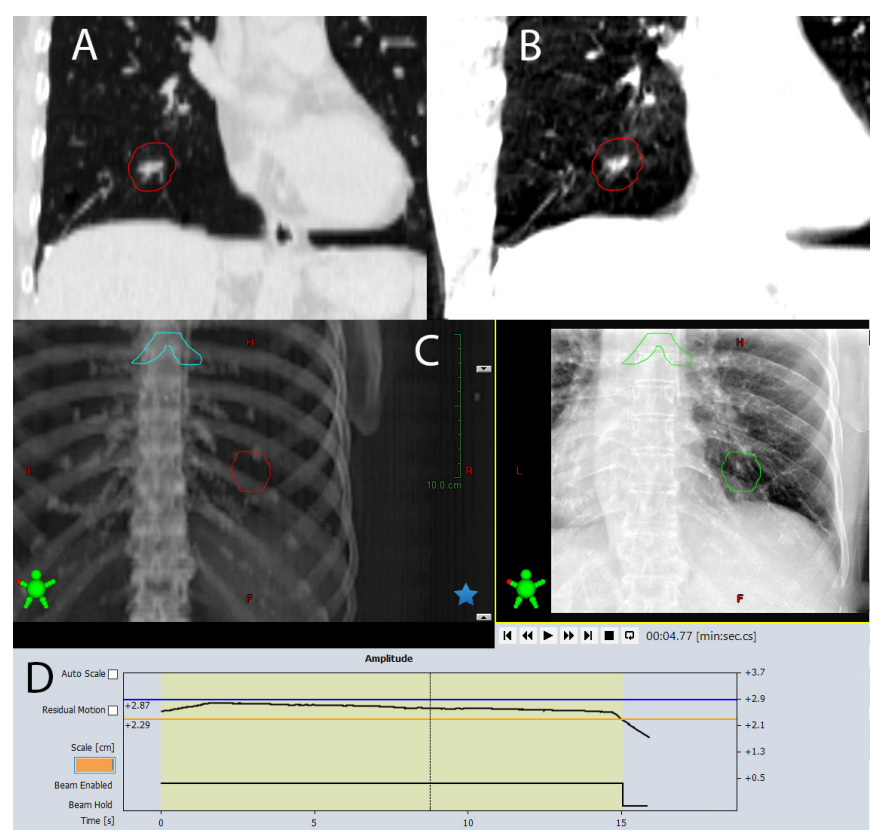

Figure 2 Images obtained during the treatment of an isolated lung metastasis from uterine cancer with no other sites of active disease. A computed tomographic (CT) image from the treatment plan with a planning treatment volume overlaid in red $(\mathrm{A})$ is compared directly to a cone-beam CT (CBCT) image obtained at the time of treatment delivery (B) by a radiation oncologist at the time of each fraction. Live postero-anterior fluoroscopic images $(C)$ can then be examined while the patient is instructed to hold their breath to limit motion of the target and nearby normal tissues. (D) A tracing of chest wall motion during both immediate pretreatment imaging and treatment delivery to ensure that the motion is within the expected excursion (black line of tracing within the lower limit (orange line) and upper limit (blue line) during $15 \mathrm{~s}$ of treatment delivery).

at 5 years and $6.8 \%$ genitourinary, $8.5 \%$ gastrointestinal, and $5.7 \%$ vaginal toxicity, comparing favorably with SBRT studies. ${ }^{5}$

The role of SBRT as a boost to a primary tumor has also been studied in a few prospective trials. A phase I dose escalation trial from Japan including patients with stage IB1-IIIB cervical cancer ineligible for intracavitary brachytherapy treated with SBRT boost suggested 22.5Gy in three fractions to be a safe regimen, although this dose is significantly lower than that routinely achieved with brachytherapy. ${ }^{6}$ A phase II trial involving SBRT boost as an alternative to brachytherapy for patients with locally advanced cervical cancer demonstrated sub-optimal outcomes with a high rate of toxicity. ${ }^{7}$ In this trial published by Albuquerque et al, patients were enrolled if they had declined brachytherapy, were deemed medically unfit to undergo the procedure, or would have required interstitial brachytherapy due to tumor extent but instead elected to forego this and enroll on trial. The 2-year local control was $70 \%$ and 2-year overall survival was $53 \%$, with rates of grade 3 or higher late gastrointestinal toxicity of $27 \%$ at 2 years. The study closed early (15/21 patients received treatment) due to poorer than expected toxicity outcomes for patients with large tumor volume $(>95 \mathrm{~mL})$. The authors further concluded that SBRT may be useful in patients with small cervical tumors (approximately $<95 \mathrm{~mL}$ ). In general, the use of SBRT for primary gynecologic tumors in lieu of 


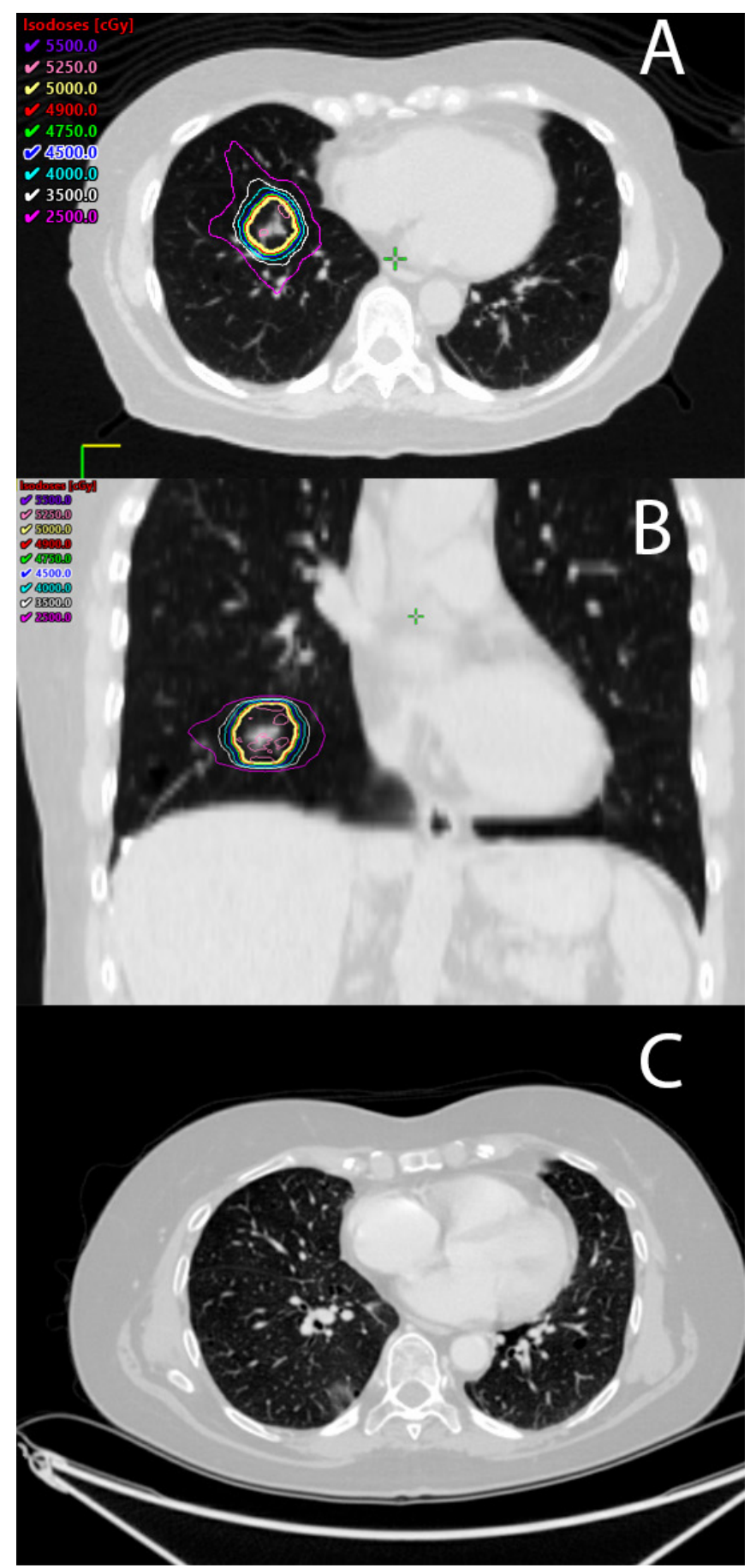

Figure 3 Clinical example of stereotactic body radiotherapy (SBRT) used to treat a solitary lung metastasis from recurrent and metastatic uterine cancer. At the time of treatment the patient had undergone systemic therapy and a single lung nodule was the only site of disease on imaging, which had demonstrated progression on multiple prior chest computed tomographic (CT) images. A treatment plan was developed to deliver 50 Gy in five fractions over 2 weeks (axial image (A) and coronal image (B) of the SBRT plan). The patient tolerated this course with no symptoms. (C) On the follow-up CT at 6 months, the pulmonary nodule was no longer visible and no new sites of disease were seen. She remained asymptomatic at last follow-up 1 year after SBRT. brachytherapy (when available) is not recommended due to these sub-optimal results unless all efforts to use brachytherapy have been exhausted. $^{8}$

There is limited information on the use of SBRT in the setting of recurrent tumors that have previously been irradiated. In a study that reported five cases of locally recurrent gynecologic cancers who received SBRT following definitive radiation therapy, there were no grade 3 toxicities reported and at least temporary local control was achieved (6 months at shortest and 19 months at longest). ${ }^{9}$ There is, however, a lack of data, both retrospectively and prospectively, that specifically investigates the role of SBRT at the primary site for locally recurrent gynecologic cancers following definitive radiotherapy, likely due to the heterogenous nature of these scenarios. As optimal external beam and brachytherapy are designed to deliver a high dose of radiation directly to the cervix, treating a recurrence with additional (and often less) radiation is not likely to result in a better outcome. Thus, primary surgical resection with consideration of intra-operative radiation therapy is a preferred approach for these cases. However, given the improvement of pelvic control with modern upfront radiotherapy techniques, including image-guided brachytherapy, these cases may become more infrequent.

However, in a parallel RetroEMBRACE observational study of image-guided brachytherapy, 222 of 731 patients developed treatment failure, with $57 \%$ being distant failure alone and $23 \%$ having both pelvic and distant failure. ${ }^{10}$ The authors conclude that, while excellent outcomes for primary disease control are achievable, there is a need for improved management of distant disease in order to optimize patient survival.

\section{SBRT AND THE OLIGOMETASTATIC STATE}

Oligometastatic disease is a term that was coined in the 1990s by Weichselbaum and Hellmann, referring to a limited number (generally $<5$ sites) of metastatic disease. ${ }^{11}$ They posit that there is a favorable sub-group of patients with metastatic disease that may benefit from localized metastases-directed ablative therapy, including surgical resection ${ }^{1213}$ and SBRT. ${ }^{14-16}$

The use of radiation therapy directed to all sites of metastatic disease has been explored in colorectal, ${ }^{17}$ lung, ${ }^{18}$ and breast cancers, ${ }^{19}$ with long-term survival rates ranging between $15 \%$ and $30 \%$. In 2014, the phase I NRG-BR001 clinical trial began enrolling patients with breast, prostate, or non-small cell lung cancer and $\leq 4$ metastases. In the 35 patients included in the results of the trial, there was no dose-limiting toxicity. ${ }^{20}$ The safety of SBRT in lung ${ }^{21}$ and liver metastase ${ }^{22}$ has also been established in multi-center phase I/II trials, with 2-year local control of $96 \%$ and $92 \%$ of the treated lung and liver metastatic lesions, respectively. A randomized phase $I / / I I$ trial of the use of ablative radiotherapy and/or surgical resection in breast cancer metastases, NRG BR002, opened in 2014 and data reporting is still in process. The SABR-COMET trial randomized 99 patients across multiple solid tumor sites with 1-4 metastases to ablative radiotherapy versus no radiotherapy. Of note, only two patients with primary gynecologic primaries were included. In this trial, systemic therapy was acceptable at the discretion of the prescribing medical oncologist and/or multi-disciplinary tumor board's recommendation in both arms of the randomization. The results of this trial revealed a trend 
Table 1 Studies using stereotactic body radiotherapy (SBRT) for primary or centrally recurrent disease in the pelvis

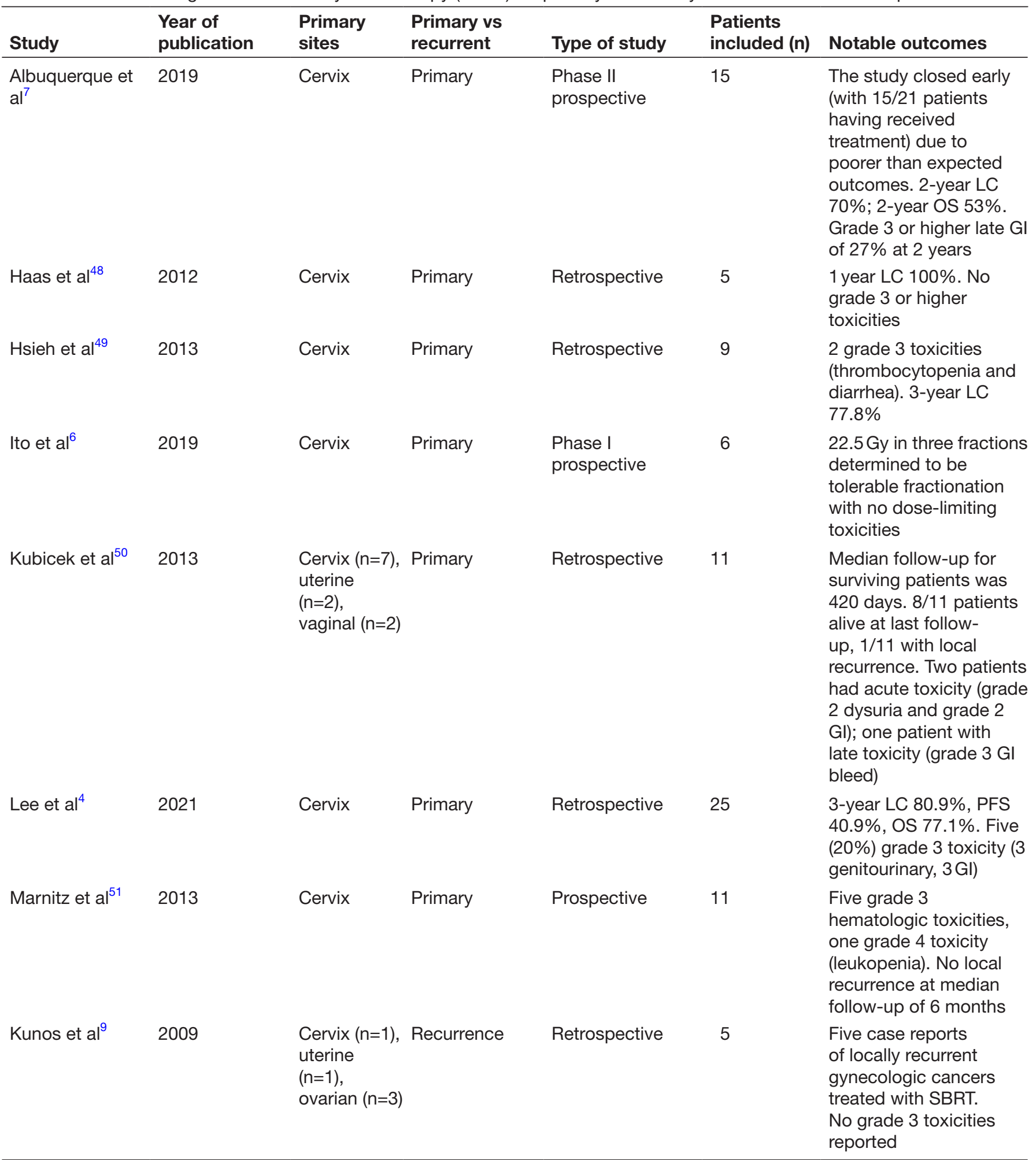

BED, biologic effective dose; GI, gastrointestinal; LC, local control; OS, overall survival; PFS, progression-free survival; SBRT, stereotactic body radiotherapy.

towards improved overall survival ( $\mathrm{HR} 0.57(95 \% \mathrm{Cl} 0.30$ to 1.10$)$, $\mathrm{p}=0.09$ ) and disease-free survival ( $\mathrm{HR} 0.47(95 \% \mathrm{Cl} 0.30$ to 0.76$)$, $\mathrm{p}=0.0012){ }^{15}$ In the long-term results of this trial, the difference in overall survival was more pronounced than the initial analysis at $42.3 \%(95 \% \mathrm{Cl} 6 \%$ to $34 \%)$ in the SABR arm and $17.7 \%(95 \%$
$\mathrm{Cl} 28 \%$ to $56 \%$ ) in the control arm (stratified log-rank $\mathrm{p}=0.006$ ). ${ }^{23}$ The phase II ORIOLE randomized trial of SABR versus observation in oligometastatic prostate cancer found similar results of improved progression-free survival (PFS) (HR 0.30 (95\% Cl 0.11 to 0.81$), p=0.002)$ in the ablative therapy arm. ${ }^{24}$ An example of a 


\section{Review}

treatment plan for lung metastases treated with SBRT is shown in Figure 2.

The use of systemic therapy with consolidative or adjuvant local therapy has also been investigated in randomized trials. The EORTC 40004 phase II trial randomized patients with liver metastases to systemic therapy with radiofrequency ablation to the metastatic lesions versus systemic therapy alone. The results of this study showed improved PFS (HR $0.57(95 \% \mathrm{Cl} 0.38$ to 0.85$), \mathrm{p}=0.005)$ and overall survival ( $\mathrm{HR} 0.58(95 \% \mathrm{Cl} 0.38$ to 0.88$), \mathrm{p}=0.01)$ in the systemic therapy and radiofrequency ablation group. ${ }^{25}$ In another phase II study investigating the use of consolidative radiotherapy therapy in non-small cell lung cancer with $\leq 3$ metastatic lesions, long-term data showed improved PFS (14.2 months with consolidative therapy vs 4.4 months with maintenance therapy or observation, $p=0.022)$ and overall survival (37.6 months with consolidative therapy vs 9.4 months, $p=0.034$ ), compared with patients on maintenance systemic therapy alone. ${ }^{26}$

\section{SBRT IN GYNECOLOGIC OLIGOMETASTASES}

The role of SBRT in treating gynecologic oligometastatic disease has not yet been clearly defined. There are minimal published prospective studies investigating the role of SBRT in oligometastatic gynecologic malignancies (Table 2). Despite this lack of data, a recent survey published in 2020 of an international group of radiation oncologists regarding the use of SBRT in gynecologic malignancies indicated that clinicians would consider SBRT for nodal $(81 \%)$ or primary recurrent disease $(91 \%) .{ }^{27}$ An important consideration when reviewing this literature is acknowledging variations in tumor biology and patterns of disease among gynecologic cancers. For example, primary ovarian cancers typically spread via intra-peritoneal shedding while cervical cancer tends to spread by direct extension. ${ }^{28}$ However, following treatment of a localized cervical cancer, patterns of failure tend to favor distant metastases (approximately 50\% following standard treatment). ${ }^{29}$ In a Korean retrospective study including over 1300 patients with primary cervical cancer investigating patterns of treatment failure, of those with recurrence $59.5 \%$ were distant while $21.5 \%$ recurred both locally and distantly. ${ }^{30}$ The recently reported RetroEMBRACE observational study found a similar pattern in the 222 recurrences among 731 women treated; $57 \%$ experienced distant recurrences and $23 \%$ had both pelvic and distant components. ${ }^{9}$ The authors conclude that, while excellent outcomes for primary disease control are achievable, there is a need for improved management of distant disease in order to optimize patient survival.

There is also a potentially curable sub-set of patients with metastatic cervical cancer to the supraclavicular lymph node region. Retrospective studies have analyzed survival outcomes for this specific patient population. In two Korean studies including women with metastatic cervical cancer to the supraclavicular nodes, 3-year survival was reported to be between $49 \%$ and $67 \% .{ }^{31}{ }^{32}$ A study from Washington University in St Louis in 24 women with supraclavicular disease treated with definitive chemoradiation found 5-year survival of $22 \% .^{33}$ In these studies, patients received definitive doses of radiotherapy to the pelvis and involved supraclavicular nodes with concurrent chemotherapy. However, these series used conventionally fractionated radiation and the role for SBRT in this clinical scenario is not well defined.

There are a few retrospective studies that investigated outcomes of ovarian oligometastatic disease. In one study reporting 82 patients diagnosed with ovarian cancer with 156 metastatic lesions treated with SBRT, control of the treated lesions at 2 years was found to be $68 \%$ and the median systemic treatment-free interval after SBRT was 7.4 months. ${ }^{34}$ Another retrospective study including 35 patients with oligometastatic ovarian cancer treated with SBRT yielded a 2-year lesional control of $80 \%$, with a higher dose (BED > 35 Gy) associated with improved control. ${ }^{35}$ A retrospective study including 20 patients (10 ovarian and 10 uterine) with a single metastatic site treated with SBRT revealed that patients with smaller tumors $(<50 \mathrm{~mL})$ had improved local control (5-year local control $<50 \mathrm{~mL} 100 \%$ vs $>50 \mathrm{~mL} 65 \%$; $\mathrm{p}<0.02) .{ }^{36}$

In a prospective phase II trial evaluating the efficacy of SBRT in 49 patients with metastatic gynecologic tumors, there was a $96 \%$ response rate in the treated lesions with a median PFS of 7.8 months and overall survival of 20.2 months. ${ }^{37}$ This was followed by the phase I trial by Kunos et al in which carboplatin and gemcitabine were delivered concurrently with SBRT to 12 patients, demonstrating that the combination therapy was tolerable but with notable grade $3(n=10)$ and $4(n=2)$ toxicities including neutropenia and hypokalemia, although this is likely more attributable to chemotherapy than to SBRT. ${ }^{38}$ There are a number of ongoing studies exploring the safety and efficacy of SBRT in oligometastatic gynecologic cancer including a phase I trial (NCT03325634) of patients with recurrent ovarian or uterine cancer with $\leq 3$ active sites of disease and a recently closed phase I trial (NCT0258293) reporting feasibility of MRI-guided SBRT to $\leq 3$ sites of metastatic ovarian cancer.

A 2017 systematic review by Mendez et al outlined the data regarding the role of radiotherapy for gynecologic malignancies and concluded that local control and toxicity seemed to be acceptable with SBRT to both primary and metastatic tumors. ${ }^{39}$ A total of 189 patients in six studies had pelvic and para-aortic lymph nodes treated with SABR with a combined local control of $83 \%$ and grade $3-4$ gastrointestinal toxicity in $3.8 \%$ of patients. ${ }^{40-45}$ In a recent systematic review article by Yegya-Raman et al, the authors compiled and analyzed 16 unique studies, either retrospective or prospective, that included SBRT for oligometastases from gynecologic origin. ${ }^{28}$ Six hundred and sixty-seven patients and more than 1000 metastatic lesions were identified, with the most common primary site being ovarian (57.6\%). Overall, the authors noted that local control ranged from $70 \%$ to $100 \%$ in studies that reported outcomes, and that disease progression often occurred outside the SBRT field. Of the studies included, 12 allowed for regional nodal metastases to be included as a site of oligometastatic disease and, of the sites treated, $64 \%$ were nodal metastases. There appeared to be a range (2-83\%) of grade 3 toxicities in seven of the 16 included studies, 373840414647 as well as four grade 4 toxicities (ranging from $2 \%$ to $8 \%$ ) in three of the reported studies. ${ }^{37} 3840$ Grade 3 toxicities included gastrointestinal (duodenal ulcer, esophagitis, and diarrhea), hematologic (neutropenia, thrombocytopenia, and leukopenia), and genitourinary (hemorrhagic cystitis), and grade 4 toxicities included neutropenia, hypokalemia, and hyperbilirubinemia. The use of concurrent chemotherapy in several of these studies is 
Table 2 Studies using stereotactic body radiotherapy (SBRT) for limited distant metastatic disease

\begin{tabular}{|c|c|c|c|c|c|}
\hline Study & $\begin{array}{l}\text { Year of } \\
\text { publication }\end{array}$ & Primary sites & Type of study & $\begin{array}{l}\text { Patients } \\
\text { included (n) }\end{array}$ & Notable outcomes \\
\hline \multicolumn{6}{|l|}{ Ovarian } \\
\hline Aghdam et $\mathrm{al}^{36}$ & 2020 & $\begin{array}{l}\text { Ovarian and } \\
\text { uterine }\end{array}$ & Retrospective & 20 & $\begin{array}{l}\text { Smaller tumors }\left(<50 \mathrm{~cm}^{3}\right) \\
\text { had improved LC }(5 \text {-year } \\
\text { LC for small vs large } \\
\text { volume } 100 \%(p<0.01) \\
\text { and } 65 \%(p<0.02))\end{array}$ \\
\hline Kowalchuk et al ${ }^{35}$ & 2020 & Ovarian & Retrospective & 35 & $\begin{array}{l}\text { 2-year LC of } 80 \% \text { with } \\
\text { a BED }>35 \text { Gy to be } \\
\text { predictive of improved LC }\end{array}$ \\
\hline \multicolumn{6}{|l|}{ Cervix } \\
\hline Hou et $\left.a\right|^{52}$ & 2019 & Cervix & Retrospective & 19 & $\begin{array}{l}\text { 2-year PFS 37\%, 2-year } \\
\text { OS } 77 \%\end{array}$ \\
\hline Ning et $\mathrm{al}^{46}$ & 2018 & Cervix & Retrospective & 38 & $\begin{array}{l}\text { 2-year PFS } 48 \%, 2-y e a r \\
\text { OS } 74 \% . \text { One grade } 3 \\
\text { toxicity (esophagitis) }\end{array}$ \\
\hline Park et $\mathrm{al}^{40}$ & 2015 & Cervix & Retrospective & 85 & $\begin{array}{l}\text { 2-year PFS } 43 \% \text {, 2-year } \\
\text { OS } 58 \% \text {. Two late grade } \\
4 \text { toxicities (rectovaginal } \\
\text { fistula) }\end{array}$ \\
\hline Kunos et $\mathrm{al}^{38}$ & 2015 & $\begin{array}{l}\text { Ovarian } \\
(n=7) \text {, uterine } \\
(n=4), \text { primary } \\
\text { peritoneal }(n=1)\end{array}$ & Phase I & 12 & $\begin{array}{l}\text { Safety of concurrent } \\
\text { chemotherapy } \\
\text { (carboplatin/gemcitabine) } \\
\text { with concurrent SBRT. } \\
\text { Grade } 4 \text { neutropenia } \\
(n=1), \text { hyperkalemia }(n=1)\end{array}$ \\
\hline Mesko et $\mathrm{al}^{47}$ & 2017 & $\begin{array}{l}\text { Ovarian } \\
(n=15), \text { uterine } \\
\text { carcinoma }(n=8), \\
\text { carcinosarcoma } \\
(n=1) \text {, cervix } \\
(n=2) \text {, vagina } \\
(n=2)\end{array}$ & Retrospective & 28 & $\begin{array}{l}\text { Favorable vs unfavorable } \\
\text { response associated } \\
\text { with tumor size }(17.2 \text { vs } \\
57.6 \mathrm{~mm}, \mathrm{p}=0.0044) \text { and } \\
\text { BED }(79.0 \text { vs } 59.6 \mathrm{~Gy} \text {, } \\
\mathrm{p}=0.027) . \text { One grade } 3 \\
\text { toxicity }\end{array}$ \\
\hline Onal et $\mathrm{al}^{53}$ & 2020 & $\begin{array}{l}\text { Ovarian }(n=8) \\
\text { cervix }(n=21)\end{array}$ & Retrospective & 29 & $\begin{array}{l}1 \text { - and } 2 \text {-year local control } \\
\text { rates for all patients } \\
\text { were } 84 \% \text { and } 84 \% \text {, } \\
\text { respectively. Complete } \\
\text { response after SBRT were } \\
\text { prognostic for survival }\end{array}$ \\
\hline
\end{tabular}


Table 2 Continued

\begin{tabular}{|c|c|c|c|c|c|}
\hline Study & $\begin{array}{l}\text { Year of } \\
\text { publication }\end{array}$ & Primary sites & Type of study & $\begin{array}{l}\text { Patients } \\
\text { included (n) }\end{array}$ & Notable outcomes \\
\hline Seo et $\mathrm{al}^{41}$ & 2015 & $\begin{array}{l}\text { Mixed population } \\
\text { with PA nodal } \\
\text { oligometastases } \\
\text { ( } 52 / 88 \text { of } \\
\text { gynecologic } \\
\text { origin) }\end{array}$ & Retrospective & 88 & $\begin{array}{l}\text { 5-year LC 92\%; 5-year } \\
\text { OS 52\% }\end{array}$ \\
\hline
\end{tabular}

BED, biologic effective dose; LC, local control; OS, overall survival; PA, para-aortic; PFS, progression-free survival; SBRT, stereotactic body radiotherapy.

likely responsible for the hematologic and hypokalemia toxicities seen, given the small irradiated volumes characteristic of SBRT. ${ }^{37} 38$

\section{SBRT, SYSTEMIC THERAPY, AND IMMUNOTHERAPY}

A major shift in the treatment paradigm within oncology is the use of immunotherapy in addition to other systemic therapy and radiation therapy. A synergistic effect between SBRT and immunotherapy may be possible, although it is not yet proven clinically. It is hypothesized that release of antigens due to SBRT may enhance the efficacy of immunotherapy. Additionally, typical radiation plans for pelvic treatment may include a large volume of bone marrow within the pelvis and lumbar spine, leading to acute and late hematologic toxicities. Since SBRT uses much smaller irradiated volumes, these techniques may be less immune suppressive and allow for better immune system activation when combined with these agents.

There are now a number of ongoing clinical trials that are aiming to investigate the safety and response rate of targeted agents and immunotherapy in combination with SBRT. A phase I trial (NCT03452332) will assess the safety of SBRT in addition to tremelimumab and durvalumab in the treatment of $\leq 2$ oligometastases from cervical, vaginal, or vulvar cancer. A phase II trial (NCT03614949) is assessing the response rate of SBRT followed by atezolizumab in $\leq 2$ oligometastases in recurrent, persistent, or metastatic cervical, vaginal, or vulvar cancer. Finally, another phase II trial (NCT03192059) is assessing the response rate of an index lesion from advanced or refractory cervical cancer, endometrial carcinoma, or uterine sarcoma plus at least one oliogmetastasis treated with an immunomodularity cocktail followed by pembrolizumab and SBRT.

\section{CONCLUSION AND FUTURE DIRECTIONS}

The utility of SBRT in the treatment of local and distant gynecologic malignancies remains an active area of investigation. It is evident that there is a body of literature that supports the use of SBRT in both the retrospective and prospective setting to achieve control of the treated site, particularly in the setting of limited metastatic disease. However, it is not recommended as an alternative to brachytherapy for intact disease unless all efforts to use brachytherapy are exhausted. While phase I and II trials have established the relative safety and potential toxicities of SBRT, there remains a dearth of phase III randomized evidence, including the use of immunotherapy, in order to better establish the role of this technique as a method of improving more global outcomes for our patients with gynecologic cancers.
Contributors All authors have contributed to the writing, editing, and review of the manuscript. All authors have reviewed the submitted paper and have approved it for submission.

Funding The authors have not declared a specific grant for this research from any funding agency in the public, commercial or not-for-profit sectors.

Competing interests None declared.

Patient consent for publication Not applicable.

Provenance and peer review Commissioned; internally peer reviewed.

ORCID iD

Junzo Chino http://orcid.org/0000-0002-3633-9685

\section{REFERENCES}

1 Timmerman RD, Herman J, Cho LC. Emergence of stereotactic body radiation therapy and its impact on current and future clinical practice. J Clin Oncol 2014;32:2847-54.

2 Nagata Y. Stereotactic body radiation therapy: principles and practices, 2015.

3 Kamrava M, Chino JP, Beriwal S. SAbR as an alternative boost modality for cervical cancer: a cautionary exercise. Int J Radiat Oncol Biol Phys 2020;106:472-4.

4 Lee TH, Song C, Kim IA, et al. Stereotactic ablative body radiotherapy boost for cervical cancer when brachytherapy boost is not feasible. Radiat Oncol 2021;16.

5 Pötter R, Tanderup K, Schmid MP, et al. MRI-guided adaptive brachytherapy in locally advanced cervical cancer (EMBRACE-I): a multicentre prospective cohort study. Lancet Oncol 2021;22:538-47.

6 Ito K, Kito S, Nakajima Y, et al. Determining the recommended dose of stereotactic body radiotherapy boost in patients with cervical cancer who are unsuitable for intracavitary brachytherapy: a phase I dose-escalation study. Jpn J Clin Oncol 2019;49:856-61.

7 Albuquerque K, Tumati V, Lea J, et al. A phase II trial of stereotactic ablative radiation therapy as a boost for locally advanced cervical cancer. Int J Radiat Oncol Biol Phys 2020;106:464-71.

8 Chino J, Annunziata CM, Beriwal S, et al. Radiation therapy for cervical cancer: Executive summary of an ASTRO clinical practice guideline. Pract Radiat Oncol 2020;10:220-34.

9 Kunos C, Chen W, DeBernardo R, et al. Stereotactic body radiosurgery for pelvic relapse of gynecologic malignancies. Technol Cancer Res Treat 2009;8:393-400.

10 Tan L-T, Pötter R, Sturdza A, et al. Change in patterns of failure after image-guided brachytherapy for cervical cancer: analysis from the RetroEMBRACE study. Int J Radiat Oncol Biol Phys 2019;104:895-902.

11 Hellman S, Weichselbaum RR. Oligometastases. J Clin Oncol 1995;13:8-10.

12 Pastorino U, Buyse M, Friedel G, et al. Long-term results of lung metastasectomy: prognostic analyses based on 5206 cases. $J$ Thorac Cardiovasc Surg 1997;113:37-49.

13 Fong Y, Cohen AM, Fortner JG, et al. Liver resection for colorectal metastases. J Clin Oncol 1997;15:938-46.

14 Gomez DR, Blumenschein GR, Lee JJ, et al. Local consolidative therapy versus maintenance therapy or observation for patients with oligometastatic non-small-cell lung cancer without progression after first-line systemic therapy: a multicentre, randomised, controlled, phase 2 study. Lancet Oncol 2016;17:1672-82. 
15 Palma DA, Olson R, Harrow S, et al. Stereotactic ablative radiotherapy versus standard of care palliative treatment in patients with oligometastatic cancers (SABR-COMET): a randomised, phase 2, open-label trial. Lancet 2019;393:2051-8.

16 lyengar P, Wardak Z, Gerber DE, et al. Consolidative radiotherapy for limited metastatic non-small-cell lung cancer: a phase 2 randomized clinical trial. JAMA Oncol 2018;4:e173501.

17 Hoyer M, Roed H, Traberg Hansen A, Hansen T, et al. Phase II study on stereotactic body radiotherapy of colorectal metastases. Acta Oncol 2006;45:823-30.

18 Griffioen GHMJ, Toguri D, Dahele M, et al. Radical treatment of synchronous oligometastatic non-small cell lung carcinoma (NSCLC): patient outcomes and prognostic factors. Lung Cancer 2013;82:95-102.

19 Milano MT, Zhang H, Metcalfe SK, et al. Oligometastatic breast cancer treated with curative-intent stereotactic body radiation therapy. Breast Cancer Res Treat 2009;115:601-8.

20 Chmura S, Winter KA, Robinson C, et al. Evaluation of safety of stereotactic body radiotherapy for the treatment of patients with multiple metastases: findings from the NRG-BR001 phase 1 trial. JAMA Oncol 2021;7:845-52.

21 Rusthoven KE, Kavanagh BD, Burri SH, et al. Multi-institutional phase I/II trial of stereotactic body radiation therapy for lung metastases. J Clin Oncol 2009;27:1579-84.

22 Rusthoven KE, Kavanagh BD, Cardenes $\mathrm{H}$, et al. Multi-institutional phase I/II trial of stereotactic body radiation therapy for liver metastases. J Clin Oncol 2009;27:1572-8.

23 Palma DA, Olson R, Harrow S, et al. Stereotactic ablative radiotherapy for the comprehensive treatment of oligometastatic cancers: long-term results of the SABR-COMET phase II randomized trial. J Clin Oncol 2020;38:2830-8.

24 Phillips R, Shi WY, Deek M, et al. Outcomes of observation vs stereotactic ablative radiation for oligometastatic prostate cancer: the ORIOLE phase 2 randomized clinical trial. JAMA Oncol 2020;6:650-9.

25 Ruers T, Van Coevorden F, Punt CJA, et al. Local treatment of unresectable colorectal liver metastases: results of a randomized phase II trial. J Natl Cancer Inst 2017;109. doi:10.1093/jnci/djx015. [Epub ahead of print: 01 Sep 2017].

26 Gomez DR, Tang C, Zhang J, et al. Local consolidative therapy vs. maintenance therapy or observation for patients with oligometastatic non-small-cell lung cancer: long-term results of a multi-institutional, phase II, randomized study. J Clin Oncol 2019;37:1558-65.

27 Leung E, Gladwish A, Sahgal A, et al. Survey of current practices from an international task force for gynecological stereotactic ablative radiotherapy. Radiat Oncol 2020;15.

28 Yegya-Raman N, Cao CD, Hathout L, et al. Stereotactic body radiation therapy for oligometastatic gynecologic malignancies: a systematic review. Gynecol Oncol 2020;159:573-80.

29 Tewari KS, Sill MW, Long HJ, et al. Improved survival with bevacizumab in advanced cervical cancer. $N$ Engl $J$ Med 2014;370:734-43.

$30 \mathrm{Kim}$ TH, Kim M-H, Kim B-J, et al. Prognostic importance of the site of recurrence in patients with metastatic recurrent cervical cancer. Int J Radiat Oncol Biol Phys 2017;98:1124-31.

$31 \mathrm{Kim}$ J-Y, Kim J-Y, Kim JH, et al. Curative chemoradiotherapy in patients with stage IVb cervical cancer presenting with paraortic and left supraclavicular lymph node metastases. Int J Radiat Oncol Biol Phys 2012;84:741-7.

32 Lee SH, Lee SH, Lee KC, et al. Radiation therapy with chemotherapy for patients with cervical cancer and supraclavicular lymph node involvement. J Gynecol Oncol 2012;23:159-67.

33 Zighelboim I, Taylor NP, Powell MA, et al. Outcomes in 24 selected patients with stage IVb cervical cancer and excellent performance status treated with radiotherapy and chemotherapy. Radiat Med 2006;24:625-30.

34 Lazzari R, Ronchi S, Gandini S, et al. Stereotactic body radiation therapy for oligometastatic ovarian cancer: a step toward a drug holiday. Int J Radiat Oncol Biol Phys 2018;101:650-60.
35 Kowalchuk RO, Waters MR, Richardson KM, et al. Stereotactic body radiation therapy in the treatment of ovarian cancer. Radiat Oncol 2020;15.

36 Aghdam N, Repka MC, McGunigal M, et al. Stereotactic body radiation therapy: a versatile, well-tolerated, and effective treatment option for extracranial metastases from primary ovarian and uterine cancer. Front Oncol 2020;10:572564.

37 Kunos CA, Brindle J, Waggoner S, et al. Phase II clinical trial of robotic stereotactic body radiosurgery for metastatic gynecologic malignancies. Front Oncol 2012;2:181.

38 Kunos CA, Sherertz TM, Mislmani M, et al. Phase I trial of carboplatin and gemcitabine chemotherapy and stereotactic ablative radiosurgery for the palliative treatment of persistent or recurrent gynecologic cancer. Front Oncol 2015;5:126.

39 Mendez LC, Leung E, Cheung P, et al. The role of stereotactic ablative body radiotherapy in gynaecological cancers: a systematic review. Clin Oncol 2017;29:378-84.

40 Park HJ, Chang AR, Seo Y, et al. Stereotactic body radiotherapy for recurrent or oligometastatic uterine cervix cancer: a cooperative study of the Korean Radiation Oncology Group (KROG 14-11). Anticancer Res 2015;35:5103-10.

41 Seo Y-S, Kim M-S, Cho C-K, et al. Stereotactic body radiotherapy for oligometastases confined to the para-aortic region: clinical outcomes and the significance of radiotherapy field and dose. Cancer Invest 2015;33:180-7.

42 Choi CW, Cho CK, Yoo SY, et al. Image-guided stereotactic body radiation therapy in patients with isolated para-aortic lymph node metastases from uterine cervical and corpus cancer. Int J Radiat Oncol Biol Phys 2009;74:147-53.

43 Bonomo P, Cipressi S, Saieva C, et al. Clinical outcome of stereotactic body radiotherapy for abdominal lymph node metastases. Tumori 2013;99:611-6.

44 Deodato F, Macchia G, Grimaldi L, et al. Stereotactic radiotherapy in recurrent gynecological cancer: a case series. Oncol Rep 2009;22:415-9.

45 Macchia G, Lazzari R, Colombo N, et al. A large, multicenter, retrospective study on efficacy and safety of stereotactic body radiotherapy (SBRT) in oligometastatic ovarian cancer (MITO RT1 study): a collaboration of MITO, AIRO GYN, and MaNGO groups. Oncologist 2020;25:e311-20.

46 Ning MS, Ahobila V, Jhingran A, et al. Outcomes and patterns of relapse after definitive radiation therapy for oligometastatic cervical cancer. Gynecol Oncol 2018;148:132-8.

47 Mesko S, Sandler K, Cohen J, et al. Clinical outcomes for stereotactic ablative radiotherapy in oligometastatic and oligoprogressive gynecological malignancies. Int J Gynecol Cancer 2017;27:403-8.

48 Haas JA, Witten MR, Clancey O, et al. CyberKnife boost for patients with cervical cancer unable to undergo brachytherapy. Front Oncol 2012;2:25.

49 Hsieh C-H, Tien H-J, Hsiao S-M, et al. Stereotactic body radiation therapy via helical tomotherapy to replace brachytherapy for brachytherapy-unsuitable cervical cancer patients - a preliminary result. Onco Targets Ther 2013;6:59-66.

50 Kubicek GJ, Xue J, Xu Q, et al. Stereotactic body radiotherapy as an alternative to brachytherapy in gynecologic cancer. Biomed Res Int 2013;2013:898953-

51 Marnitz S, Köhler C, Budach V, et al. Brachytherapy-emulating robotic radiosurgery in patients with cervical carcinoma. Radiat Oncol 2013;8:109.

52 Hou X, Wang W, Zhang F, et al. Stereotactic body radiation therapy for oligometastatic pulmonary tumors from cervical cancer. Asia Pac $J$ Clin Oncol 2019;15:e175-80.

53 Onal C, Gultekin M, Oymak E, et al. Stereotactic radiotherapy in patients with oligometastatic or oligoprogressive gynecological malignancies: a multi-institutional analysis. Int J Gynecol Cancer 2020;30:865-72. 